\title{
Robotic totally endoscopic coronary artery bypass grafting (TECAB) - placement of bilateral internal mammary arteries to the left ventricle
}

\author{
Johannes Bonatti ${ }^{1}$, Antti Vento ${ }^{2}$, Nikolaos Bonaros ${ }^{3}$, Mahmoud Traina $^{1}$, Eric Lehr ${ }^{4}$ \\ ${ }^{1}$ Heart and Vascular Institute, Cleveland Clinic Abu Dhabi, Abu Dhabi, United Arab Emirates; ${ }^{2}$ HUH Heart and Lung Center, University Hospital, \\ Helsinki, Finland; ${ }^{3}$ University Clinic of Heart Surgery, Innsbruck Medical University, Innsbruck, Austria; ${ }^{4}$ Swedish Heart and Vascular Institute, \\ Swedish Medical Center, Seattle, WA, USA \\ Correspondence to: Johannes Bonatti, MD, FETCS. Cardiac Surgeon, Chief Heart \& Vascular Institute, Cleveland Clinic Abu Dhabi, PO Box 112412, \\ Abu Dhabi, United Arab Emirates. Email: bonattj@clevelandclinicabudhabi.ae.
}

Submitted Aug 30, 2016. Accepted for publication Oct 14, 2016.

doi: $10.21037 /$ acs.2016.11.05

View this article at: http://dx.doi.org/10.21037/acs.2016.11.05

\section{Clinical vignette}

Totally endoscopic coronary artery bypass grafting (TECAB) to date can only be carried out in a reproducible manner using robotic technology. The procedure has evolved from single left internal mammary artery (LIMA) bypass grafting of the left anterior descending (LAD) artery to complex multivessel surgical revascularization $(1,2)$. The main advantage of an endoscopic approach is reduced surgical trauma and earlier recovery. Bilateral internal mammary arteries have demonstrated superior long-term survival after multivessel coronary artery bypass grafting $(3,4)$. A specific advantage of the technique shown in this surgical technical paper and video is placement of bilateral internal mammary arteries to the left ventricle without splitting the sternum. In our practice, the arrested heart technique has been proven to be very helpful in developing multivessel TECAB. By fully unloading the heart, operative space is maximised due to the fact that both lungs can be deflated, and all coronary artery territories can be reached.

\section{Surgical techniques}

The surgical technique is shown in the video (Video 1).

\section{Preparation}

Anesthesia follows general cardiac anesthesia principles but some specifics require attention. Patients require double lumen endotracheal tube intubation or a bronchial blocker for single lung ventilation. Percutaneous defibrillator patches and cerebral near infrared spectroscopy (NIRS) leads are placed for cerebral perfusion and leg perfusion monitoring. A pulmonary artery vent is inserted for support of venous drainage. Transesophageal echocardiography (TEE) is mandatory throughout the whole procedure to adequately monitor heart function and position of the endo-aortic occlusion balloon. For monitoring of the endoballoon bilateral radial artery pressure lines are inserted as well.

\section{Exposition}

The patient is placed on the operating table in the supine position with the arms tucked to the body and the left chest elevated to approximately 30 degrees using a towel roll.

Ports are introduced on the patient's left chest and should be inserted by the most experienced team member, as correct port placement plays a key role in the operation. Insertion requires complete left lung collapse that should be confirmed by the anesthesiologist before placement. A camera port is placed in the fourth intercostal space along the anterior axillary line and $\mathrm{CO}_{2}$ is insufflated at a pressure of $8 \mathrm{mmHg}$. After inspection of the thoracic cavity with the robotic camera, the left and right instrument ports are inserted cranially and caudally four fingerbreadths away from the camera port, slightly lateral to the mid-clavicular line. The robotic arms are then docked to the ports. 
Currently we use the da Vinci Si model (Intuitive Surgical, Sunnyvale, CA, USA).

\section{Operation}

\section{Internal mammary artery harvesting}

Using long-tip forceps on the left and electrocautery on the right instrument arm, a retrosternal dissection is carried out and the right pleural space is entered. These maneuvers are performed using "camera up" view with the 30 degree angled robotic camera. The right internal mammary artery (RIMA) is identified by its visible pulsations. Electrocautery is set at 15 to 20 watts depending on the brand of electrosurgical units used and the endothoracic fascia and muscle covering the internal mammary artery are removed. The RIMA is then harvested in a skeletonized fashion, gently teasing the artery from the chest wall with the cautery spatula while cauterizing side branches close to the chest wall. Clipping is necessary only for large branches and in case of side branch bleeding. After heparinization, the internal mammary artery can be clipped distally, divided using robotic Pott's scissors and dropped into the left chest for autodilatation. The RIMA is harvested first, followed by harvesting of the LIMA. Technique and time requirements are the same.

\section{Assistance port placement}

After internal mammary artery harvest, an $8 \mathrm{~mm}$ assistance port is inserted opposite the camera port parasternal. This port allows for introduction and removal of suture material, bulldog clamps, and other necessary materials. A 12-mm subcostal port is introduced two fingerbreadths from the xiphoid process to the left and docked to the fourth arm of the robotic system.

\section{Cannulation, initiation of cardiopulmonary bypass, and induction of cardioplegic arrest}

The left groin vessels are exposed simultaneously to RIMA and LIMA harvesting. We keep dissection of the femoral artery and vein limited in order to prevent lymphatic leaks. A leg perfusion catheter is inserted in all cases and leg perfusion is monitored by NIRS throughout the case. Venous drainage is accomplished through a $25 \mathrm{Fr}$ venous drainage cannula advanced into the superior vena cava under TEE guidance. A $21 \mathrm{Fr}$ or $23 \mathrm{Fr}$ arterial perfusion cannula with a side arm is inserted into the femoral artery and connected to the arterial line of the cardiopulmonary bypass circuit.
The endo-aortic balloon catheter is prepared by the scrub team and inserted through the side arm of the arterial perfusion cannula. A 0.035 -inch guide wire is brought through the balloon catheter into the ascending aorta under TEE guidance. Once its position just above the aortic valve is confirmed, the endo-balloon is advanced into the aortic root. The guide wire is then pulled back and the endoballoon catheter is connected to the heart lung machine tubing. Pressure lines for monitoring of balloon pressure and aortic root pressure are de-aired and connected to corresponding manometers.

Cardiopulmonary bypass is slowly initiated in all cases. With adequate venous drainage, low blood pressure, and confirmation of lack of ventricular ejection the endo balloon is inflated and its correct position in the aortic root is confirmed by TEE. Having confirmed adequate position and inflation of the balloon, adenosine $(6 \mathrm{mg}$ diluted in $20 \mathrm{~mL}$ of normal saline solution) is then injected into the aortic root, which usually leads to immediate asystole. Infusion of cardioplegia follows and is repeated every $20 \mathrm{~min}$.

\section{Resection of the pericardial fat pad and pericardiotomy}

All the following surgical steps are carried out with the 30-degree robotic scope view "down".

A pericardial drainage hole is created posterior to the left phrenic nerve. This step is important to avoid later accumulation of blood in the posterior pericardial sac while working on the circumflex artery branches. Using the electrocautery spatula on the right and a long-tip forceps on the left, the pericardial fat pad is mobilized, starting cranially and moving caudally. With the patient on cardiopulmonary bypass this maneuver is facilitated in patients with prominent fat pads. The pericardium is then incised longitudinally, working from cranial to caudal and "T"-ing off the incision at the caudal reflection. Cranially, the incision is carried into the direction of the left atrial appendage with caution.

\section{Construction of the coronary artery bypass anastomoses}

The EndoWrist stabilizer is inserted through a subxiphoid port, which is then docked to the fourth arm of the robotic system. This is a suction stabilizer, originally designed for beating heart TECAB, but can be perfectly used to expose the circumflex coronary artery system. The surgeon slides this instrument alongside the lateral wall of the heart and then turns on the suction. Holding the lateral wall this way allows the obtuse marginal branches and the circumflex 
coronary artery end branch to be rotated into view. Target vessels are exposed using Pott's scissors and incised with a robotic Lancet beaver knife. Small bulldog clamps are placed on the internal mammary arteries, which are prepared distally and free flow is assessed.

A video and detailed description of our suturing technique is available at http://www.youtube.com/ watch? $v=16 \mathrm{DiBz} 2 \mathrm{JUnY}$. In brief a 7/0, $7 \mathrm{~cm}$ double-armed polypropylene suture is used. We start suturing at the toe and then suture clockwise around the back wall. After going around the heel the anterior wall is completed in counterclockwise fashion. Specific attention needs to be paid to ensure adequate suture tension and robotic anastomotic suturing needs intense training in simulation models (virtual and porcine wetlab on models) before clinical application.

The endostabilizer can also be used to bring the left anterior descending (LAD) artery into a comfortable position. As shown in the video, our standard approach is to place a RIMA to the LAD in situ graft and a LIMA in situ graft to the circumflex coronary artery territory. For distal circumflex territories, a Y graft of RIMA to LIMA is constructed before opening the pericardium and commencing cardiopulmonary bypass. The anastomotic technique for the Y-graft and the distal anastomosis is as described above.

\section{Reperfusion and weaning from cardiopulmonary bypass}

It is important to remove all suture material and bulldog clamps from the operative field while the heart is still arrested. This may become difficult after balloon deflation as the heart becomes hyperdynamic. After deflation of the endo balloon the heart usually starts beating spontaneously in sinus rhythm. Endoscopic transit time ultrasound flow measurements on the grafts are carried out using flow probes inserted through the subcostal port. After confirmation of adequate hemostasis double lung ventilation is started and the patient is weaned from cardiopulmonary bypass.

\section{Completion}

The left lung is deflated again and adequate coagulation and hemostasis is confirmed. Residual blood is evacuated from the pleural spaces using an endotracheal suction tube inserted through the assistance port. All robotic instruments are then removed and the robotic system is undocked. It is important to leave the ports in place and to remove them under scope vision through the subcostal port. All port sites are thoroughly cauterized and packed with Surgicel ${ }^{\mathrm{TM}}$. A chest tube is inserted through the camera port, which should be inserted with the left lung inflated to avoid injuries to the bypass grafts. Local anesthetic is infiltrated into the port sites to support postoperative pain control.

\section{Postoperative care}

Postoperative care follows general principles of care for cardiac surgery patients.

\section{Additional aspects}

General details on TECAB indications, contraindications, and surgical technique can also be accessed at https://www. youtube.com/watch?v=IB02HOfeUdM

\section{Comments}

\section{Clinical results}

We have published our TECAB results alone and as combinations of TECAB with PCI in hybrid coronary revascularization $(1,2,5)$. Results appear to meet the standards of standard CABG through sternotomy.

\section{Advantages}

The main advantage of this technique is placement of bilateral internal mammary arteries without the trauma of sternotomy. This benefit is particularly appealing for coronary surgery patients with risk factors for deep sternal wound infection such as diabetes, obesity and chronic obstructive pulmonary disease (COPD). The arrested heart technique as compared to beating heart techniques (which are also performed by our group) offers the advantage of tremendous space gain and access to all coronary artery targets including low obtuse marginal branches and the distal circumflex coronary artery. As compared to minithoracotomy approaches on the beating heart our technique offers better visual overview and better suturing comfort. With robotic endoscopic bilateral IMA grafting a hybrid revascularization concept can be offered to patients with complex multivessel coronary artery disease (5).

\section{Caveats}

Prior to performing multivessel robotic endoscopic 
revascularization techniques, the surgeon is required to be proficient with single LIMA to LAD grafting, as well as peripheral cannulation for cardiopulmonary bypass and application of the endo-balloon. Technical suturing skills need to be practiced in simulation models. Due to the complexity of the procedure, time requirements for $\mathrm{TECAB}$ are increased as compared to operations through sternotomy.

\section{Acknowledgements}

None.

\section{Footnote}

Conflicts of Interest: The authors have no conflicts of interest to declare.

Cite this article as: Bonatti J, Vento A, Bonaros N, Traina $\mathrm{M}$, Lehr E. Robotic totally endoscopic coronary artery bypass grafting (TECAB)-placement of bilateral internal mammary arteries to the left ventricle. Ann Cardiothorac Surg 2016;5(6):589-592. doi: 10.21037/acs.2016.11.05

\section{References}

1. Bonatti J, Schachner T, Bonaros N, et al. Robotically assisted totally endoscopic coronary bypass surgery. Circulation 2011;124:236-44.

2. Bonatti J, Lee JD, Bonaros N, et al. Robotic totally endoscopic multivessel coronary artery bypass grafting: procedure development, challenges, results. Innovations (Phila) 2012;7:3-8.

3. Vallely MP, Edelman JJ, Wilson MK. Bilateral internal mammary arteries: evidence and technical considerations. Ann Cardiothorac Surg 2013;2:570-7.

4. Lytle BW. Bilateral internal thoracic artery grafting. Ann Cardiothorac Surg 2013;2:485-92.

5. Bonatti JO, Zimrin D, Lehr EJ, et al. Hybrid coronary revascularization using robotic totally endoscopic surgery: perioperative outcomes and 5-year results. Ann Thorac Surg 2012;94:1920-6; discussion 1926. 